\title{
Comparative Efficacy in Preventing Plaque Formation around Pit and Fissure Sealants: A Clinical Trial
}

\author{
Bennett T Amaechi ${ }^{1}$, Hariyali Kasundra ${ }^{2}$, Linda O Okoye ${ }^{3}$, Phat L Tran ${ }^{4}$, Ted W Reid ${ }^{5,6}$
}

\begin{abstract}
Aim: The purpose of this study is to compare the clinical performance of an organo-selenium-containing pit and fissure sealant with that of a selenium-free sealant for clinical retention and prevention of plaque and caries development around the sealants.

Materials and methods: Following an in vitro study confirming the antimicrobial effect of an organo-selenium-containing pit/fissure sealant [DenteShield $\left.{ }^{T M}(D S)\right], 120$ adolescents (7-20 years old) at varying caries risk status had DS sealant applied to a single tooth on the left or the right side of the dentition and UltraSeal ${ }^{\mathrm{TM}}$ XT Plus (UXT) on a corresponding tooth on the opposite side. Sealants' assessment was performed quarterly for 1 year for clinical retention, plaque, and caries formation around the sealant. Each sealant lost was replaced but considered as a failure in further analysis. McNemar's test was used to statistically analyze the outcome variables at each assessment time point.

Results: While $7 \%$ and $12 \%$ plaque growth was observed around the UXT sealant at 9th and 12th months, respectively, DS exhibited 100\% prevention of plaque growth. Both sealants exhibited $100 \%$ caries prevention. Clinical retention did not significantly differ between DS and UXT at all assessment time points except at 12 months when DS showed statistically significantly $(p<0.001)$ better retention $(96 \%)$ than UXT ( $81 \%)$.

Conclusion: In this study, while both sealants are equally effective in caries prevention, DS completely prevented plaque growth around it with better clinical retention than UXT that offered only limited protection against plaque growth.

Clinical significance: Being antimicrobial, DS pit and fissure sealant may be the best sealant option for patients whose caries risk status is due to poor oral hygiene.
\end{abstract}

Keywords: Caries prevention, DenteShield ${ }^{\mathrm{TM}}$, Pit and fissure sealant, UltraSeal ${ }^{\mathrm{TM}} \mathrm{XT}$ Plus ${ }^{\mathrm{TM}}$.

The Journal of Contemporary Dental Practice (2019): 10.5005/jp-journals-10024-2552

\section{INTRODUCTION}

The pits and fissures in occlusal surfaces of the first and second molars are most susceptible to dental caries among school-aged children. ${ }^{1}$ Based on the evidence on its effectiveness, sealing the occlusal surfaces of the permanent molars is a recommended procedure to prevent caries on those teeth. ${ }^{2,3}$ Sealing the occlusal surfaces using pit and fissure sealants prevents caries by eliminating plaque stagnation areas, thus preventing plaque accumulation that promotes decay in occlusal pits and fissures. . $^{3,4}$ Sealants may be indicated for children and adults with sufficiently erupted permanent teeth that are anatomically susceptible to caries, especially when the individual is at moderate or high caries risk status. ${ }^{2-6}$ Sealants may also be indicated as the treatment option for incipient caries that are limited to the enamel of pits and fissures. ${ }^{2-6}$

Commercially available pit and fissure sealant materials are of two types: resin-based and glass ionomer sealants. The most commonly used being the visible light-activated fluoridecontaining sealants. The effectiveness of resin-based sealants, which depends on the longevity of sealant coverage (i.e., clinical retention), has been demonstrated in many studies. ${ }^{7-9}$ Whether the fluoride release from sealants has any additional beneficial effects in caries prevention is still questionable. ${ }^{10}$ However, fluoridereleasing sealants may strengthen the tooth surface to increase its resistance to caries development and remineralize an underlying enamel caries lesion. ${ }^{11-13}$ Apart from lack of retention, a major cause of sealant failure or caries formation in the presence of a sealant is plaque formation around the edge of an improperly contoured sealant and underneath a leaking sealant. ${ }^{14}$ \begin{tabular}{l}
\hline \hline 1,2 Department of Comprehensive Dentistry, School of Dentistry, \\
University of Texas Health Science Center at San Antonio, San Antonio, \\
Texas, USA \\
${ }^{3}$ Department of Restorative Dentistry, Faculty of Dentistry, College of \\
Medicine, University of Nigeria Teaching Hospital, Ituku Ozalla, Enugu \\
State, Nigeria \\
${ }^{4,5}$ Department of Ophthalmology and Visual Sciences, Texas Tech \\
University Health Sciences Center, Lubbock, Texas, USA \\
${ }^{6}$ Department of Medical Microbiology and Immunology, Texas Tech \\
University Health Sciences Center, Lubbock, Texas, USA
\end{tabular}

Corresponding Author: Linda O Okoye, Department of Restorative Dentistry, Faculty of Dentistry, College of Medicine, University of Nigeria Teaching Hospital, Ituku Ozalla, Enugu State, Nigeria, Phone: +2348068424848, e-mail: linda.okoye@unn.edu.ng

How to cite this article: Amaechi BT, Kasundra H, Okoye LO, Tran PL, Reid TW. Comparative Efficacy in Preventing Plaque Formation around Pit and Fissure Sealants: A Clinical Trial. J Contemp Dent Pract 2019;20(5):531-536.

Source of support: SelenBio Inc., Austin, TX

Conflict of interest: None

Thus, the development of a sealant with antimicrobial action should be an advance in caries prevention. SelenBio Incorporated has developed an antibacterial pit and fissure sealant, DenteShield ${ }^{\mathrm{TM}}$ (DS) pit and fissure sealant (SelenBio Inc., Austin, TX), which has received FDA 510(k) clearance (\#K09059). The present clinical study assessed the effectiveness of DS pit and fissure sealant in children and adolescents by evaluating (1) clinical retention (full

(0) The Author(s). 2019 Open Access This article is distributed under the terms of the Creative Commons Attribution 4.0 International License (https://creativecommons. org/licenses/by-nc/4.0/), which permits unrestricted use, distribution, and non-commercial reproduction in any medium, provided you give appropriate credit to the original author(s) and the source, provide a link to the Creative Commons license, and indicate if changes were made. The Creative Commons Public Domain Dedication waiver (http://creativecommons.org/publicdomain/zero/1.0/) applies to the data made available in this article, unless otherwise stated. 
or partial), including mobility of the sealant; (2) plaque formation on and around the sealant (evidence of bacterial growth); (3) caries formation around the sealant; and (4) the safety of the sealant (assessed by the presence of any side effects on oral soft tissues). The effectiveness of DS was compared with that of a commonly used resin-based fluoride-releasing sealant, UltraSeal ${ }^{T M}$ XT Plus ${ }^{T M}$ (Ultradent Inc., South Jordan, UT, USA), following an in vitro study confirming the antibacterial effect of DS.

\section{Materials and Methods}

The study was performed in two phases, in vitro and in vivo studies. The in vitro study, which was conducted at the Texas Tech University Health Sciences Center at Lubbock in Texas, was an antimicrobial testing of DS and a placebo sealant against the growth of three different cariogenic bacteria, while the in vivo study, conducted at the University of Texas Health Sciences Center at San Antonio in Texas, was a clinical study comparing the clinical performance of DS with that of a selenium-free sealant, UltraSeal XT Plus ${ }^{\mathrm{TM}}$ (UXT).

\section{The In Vitro Biofilm Study: Antimicrobial Testing}

\section{Bacterial Strains, Media, and Growth Conditions}

Streptococcus salivarius strain ATCC ${ }^{\oplus} 13419$ and Streptococcus mutans strain ATCC $^{\circledR} 35668$ were obtained from Remel (Lenexa, Kansas). Streptococcus sanguis ATCC 10556 was obtained from ATCC. Streptococcus salivarius, S. sanguis, and S. mutans were routinely grown in brain heart infusion (BHI).

\section{Sealant Materials}

Both the selenium-containing sealant, DS, and a placebo sealant with the same composition as DS except selenium were obtained from SelenBio Inc., Austin, Texas. The selenium-free placebo sealant was specially manufactured for the authors by SelenBio Inc., Austin, Texas, specifically for this study, and as such it is not commercially available. Both sealants were made into $7 \mathrm{~mm}$ discs for testing of bacterial attachment.

\section{Quantitative Analysis of the Biofilms (CFU/Segment)}

The biofilm assay was done as described by Hammond et al. ${ }^{15}$ and Tran et al., ${ }^{16}$ using the microtiter plate assay with some modifications. Discs of $7 \mathrm{~mm}$ diameter were made from either the selenium-free placebo sealant or the organo-selenium sealant. To grow biofilms on each disc of both sealants, each disc was incubated in $1 \mathrm{~mL} \mathrm{BHI}$ media in the presence of approximately 1000 initial colony forming units (CFU) of S. salivarius, 1000 CFU of S. sanguis, or 7500 CFU of S. mutans in each well of the microtiter plate at $37^{\circ} \mathrm{C}$ for 24 hours. The incubation was under micro-aerobic conditions, which were generated by placing the plate inside a gas jar containing an EZ GasPak (BD, Franklin Lakes, New Jersey, Catalog no. 260678). Biofilms were quantified by determining the CFU per sealant disc. To determine the CFU per disc, each piece was carefully removed from the well, rinsed gently with sterile distilled water, and placed into a microcentrifuge tube containing $1 \mathrm{~mL}$ of phosphate-buffered saline (PBS). The tubes were placed in a water bath sonicator for a total of 10 minutes to loosen the cells within the biofilm and then vigorously vortexed three times for 1 minute to detach the cells. Suspended cells were serially diluted 10 -fold in PBS, and $10 \mu \mathrm{L}$ aliquots of each dilution were spotted onto Tryptic Soy agar with $5 \%$ sheep blood. The agar plates were incubated at $37^{\circ} \mathrm{C}$ for 24 hours and the number of CFU was counted. Following this, the quantity of biofilm per disc was determined using the following formula: number of CFU $\times$ dilution factor $\times 100$. To confirm the efficacy of our protocol for recovery of biofilm-associated bacteria from each sealant disc, the discs were washed three times with sterile PBS, placed into fresh tubes containing PBS, and the sonication and vortexing processes were repeated. We neither recovered CFU from the plating of the PBS wash after the new sonication (data not shown) nor were we able to visualize residual bacteria by scanning electron microscopy on the vortexed segments.

\section{The In Vivo Study: Clinical Trial}

\section{Study Design}

This was a randomized double-blind split-mouth study involving paired tooth surfaces. The study recruited 120 children and adolescents of age 7-20 years old, of mixed gender, from varied ethnic origins and socioeconomic status, at moderate or high caries risk status. Subjects were selected such that there was a mixture of tooth surface conditions, sound, or incipient caries limited to the enamel of pits and fissures. Every subject received DS sealant on occlusal pits and fissures of molars or premolars on either the left or the right side of the dentition and UXT on the corresponding tooth on the opposite side of the dentition. The side to receive the DS sealant on each subject was randomly selected by computer output. Pits and fissures of sealed paired teeth shared a similar health status either sound or with incipient caries limited to the enamel. The clinical status of sealants was examined every 3 months for 12 months for retention (total retention, partial, or total loss), plaque and/or caries formation around and/or underneath the sealant, and presence of any side effect. Lost sealants were reapplied but tooth was considered as a failure in the further analysis.

\section{Sample Size Calculation}

For the calculation of the sample size, comparisons involving binary outcomes measured on a categorical scale (i.e., caries formation, retention/mobility, and side effects) were tested using McNemar's procedure for paired per cents. Assuming a $5 \%$ caries incidence in the DS-sealed teeth and a 17\% caries incidence in the UXT-sealed teeth and a $1 \%$ joint caries incidence (caries in both teeth), then for a sample size of $n=100$, McNemar's test will have power equal to 0.80 under a two-sided alternative hypothesis and $a=0.05$. The target sample size of 120 allowed a $20 \%$ dropout over 12 months of study monitor.

\section{Subject Recruitment}

The study recruited 120 children and adolescents of age 7 to 20 years old, of mixed gender, different ethnic origins, varied socioeconomic status, and at moderate or high caries risk status. The caries status was assessed using American Dental Association (ADA) caries risk assessment tools and determined based on the recommendation of the ADA Council on Scientific Affairs. ${ }^{17}$ The majority of the subjects (approximately 95\%) were recruited from two elementary schools in San Antonio, where the sealants were placed in a mobile dental facility. The rest of the subjects were recruited via a flyer, and their sealants were placed at the Research Clinic of the University of Texas Health Science Center School of Dentistry. Prior to the recruitment exercise, approval was obtained from the Institutional Review Board of University of Texas Health Science Center at San Antonio (approval no. HSC20100380H), who ensured that all proper procedures to protect human subjects were 
followed. Subjects were recruited based on the following inclusion and exclusion criteria.

Besides age of 7 through 20 years, which excluded those below the age of 7 or above 20 years, subjects must be at either moderate or high caries risk status to be included while low caries risk status excludes a subject from the study. In addition to age and caries risk status, subjects were included into the study only if they (or their surrogate, if a child) were able to read and sign a written informed consent form that explains the study and have at least one existing occlusal pit or fissure in at least one fully erupted paired permanent tooth surfaces (e.g., left and right lower first molars) that were anatomically susceptible to caries. The paired permanent tooth surfaces must be in similar health status, i.e., sound or with incipient caries limited to the enamel of pits and fissures. Tooth surfaces with restoration or sealant coverage in the pit and fissure system were excluded from the study. The subject also had to agree to give a full medical and drug history, visit every 3 months for assessment, and have telephone contact for scheduling appointment and monitoring adverse effects.

\section{Study Protocol}

Following confirmation of eligibility and obtaining consent, the sealant was placed on each subject by one experienced dentist licensed in the state of Texas, and who has been involved in school sealant programs for elementary schools in San Antonio and Laredo, all in Texas. The subjects received instructions on good oral health behavior and were individually shown how to clean their teeth by trained oral health educators prior to the start of the treatment. Every subject received DS on the one side of the dentition (upper or lower quadrant) and UXT on the corresponding tooth on the opposite side, i.e., if DS was placed on the left lower first molar, then the right lower first molar received UXT. This was to eliminate the possible influence, on sealant retention, of the habit of using one side of the mouth more than the other side for food mastication, exhibited by some individuals. The side to receive the DS sealant was randomly selected by computer output. The sealed occlusal pits and fissures of the paired teeth shared similar health status, both were either sound or have incipient caries limited to the enamel. Only pits and fissures in occlusal surfaces of molars and premolars deemed to be anatomically susceptible to caries, or to have incipient caries limited to the enamel of pits and fissures, were sealed.

\section{Sealant Procedures}

Following selection and identification of the teeth to be sealed, sealants were placed in accordance with the recommended clinical procedures as described by Govoni, ${ }^{18}$ and in accordance with the sealant manufacturer's instruction. No fissurotomy was performed on incipient caries on pits and fissures prior to sealant placement.

\section{Clinical Evaluation}

The sealant was evaluated every 3 months for a total of 12 months for (1) clinical retention (full or partial), including mobility of the sealant, (2) plaque formation on and around the sealant, (3) caries formation around the sealant, and (4) safety of the sealant (assessed by the presence of any side effect on oral soft tissues). All clinical evaluation both at the elementary schools and the university clinical research facility was performed by one and the same examiner (LOO) assisted by a research assistant (HK) serving as the recorder.

\section{Retention and Mobility Evaluation}

All sealed surfaces were examined and recorded at the baseline (immediately after sealant placement). These surfaces were then evaluated at 3, 6, 9, and 12 months visit intervals. Sealant retention was evaluated using both visual and tactile techniques, i.e., in addition to the visual presence or absence of a sealant; an explorer was used to determine the mobility and marginal integrity of the sealant. The following sealant retention criteria were used: $1=$ totally present and immobile; 2 = partially lost and immobile; 3 = partially lost and mobile; and $4=$ totally lost or totally present and mobile. At any assessment visit, sealants that scored 2, 3, or 4 are replaced; however, the affected tooth was considered as a failure in the subsequent clinical assessment.

\section{Plaque Formation}

This assessment was performed using the quantitative light-induced fluorescence (QLF Pro ${ }^{\mathrm{TM}}$, Inspektor Research, Inc., Amsterdam, The Netherland) in a red fluorescence mode as described by HeinrichWeltzien et al. ${ }^{19}$ QLF Pro ${ }^{\text {TM }}$ utilizes the principle of red fluorescence emission by some bacteria/plaque to detect and monitor the presence of bacterial plaque and, hence, bacterial activities on tooth surfaces. ${ }^{19-22}$

\section{Caries Formation}

Surfaces with incipient caries in the pits and fissures prior to sealant placement were noted at the baseline (immediately after sealant placement). The development of new caries lesions in sealed pits and fissures was diagnosed as (1) incipient or (2) cavitated caries, following the current clinical criteria for caries diagnosis using the conventional visual-tactile method. No radiograph was employed.

\section{Safety (adverse/side effect) Evaluation}

Before the placement of the sealant, the subject's oral mucosa was examined for any soft tissue lesion. Following the placement of the sealant, each subject was contacted through telephone calls by the study coordinator on a monthly basis to enquire about any side or adverse effects that may relate to the sealant. Side effects were also monitored on every evaluation visit.

\section{Statistical Analysis}

For statistical evaluation of retention, scores 2, 3, and 4 were classified as a failed sealant, while score 1 was classified as successfully retained. McNemar's test, a test of paired proportions, was employed to evaluate statistical differences in retention between the paired UXT and DS sealants at each period of assessment. For statistical evaluation of the caries outcome on sealed surfaces, the percentage of DS-sealed surfaces that developed caries during the period of the study was calculated relative to the total number of DS-sealed surfaces. The percentage of UXT-sealed surfaces was calculated in a similar way. Similarly, the effectiveness of the sealants in preventing plaque formation (bacterial growth) was calculated in percentage as above. The logistic regression test was used to examine the association between sealant retention or plaque formation and other variables (gender, age, molar or premolar, upper or lower jaw, and left or right side).

\section{Results}

\section{The In Vitro Biofilm Study}

While full growth (without any inhibition) of the three bacteria strains was observed on the discs of the placebo sealant, the DS sealant resulted in a reduction of (1) 6 logs of inhibition of S. salivarius 
binding (Graph 1A); (2) 5 logs of S. Sanguis binding (Graph 1B); and (3) 5 logs of S. mutans binding (Graph 1C). In the case of both S. salivarius and S. mutans, this was complete inhibition of binding. Thus, there was statistically significant inhibition of all three of the different bacterial strains with DS when compared with the placebo sealant that offered no inhibition.

\section{The Clinical Study}

Among the 120 subjects recruited into the study, 40 were at moderate caries risk, while 80 were at high caries risk. A total of 144 pairs of fissure sealants were placed on 120 subjects ( 46 males and 74 females) at baseline. Seventy pairs of sealants were placed with DS on the left and UXT on the right, while 74 pairs had DS on the right and UXT on the left. Six subjects, with 13 pairs of sealants, withdrew from the study at the last quarter due to either change in school or relocation from San Antonio. One hundred and fourteen (114) subjects, with 131 pairs of sealants at baseline, completed the study. The overall result of the study is shown in Table 1. At the last examination after 12 months of application, $96 \%$ (126 of 131) of the DS and $81 \%$ (106 of 131) of the UXT sealants were completely retained (score 1), and this difference in retention proportion was statistically significant $(p<0.001)$. With the DS sealant, $3.0 \%$

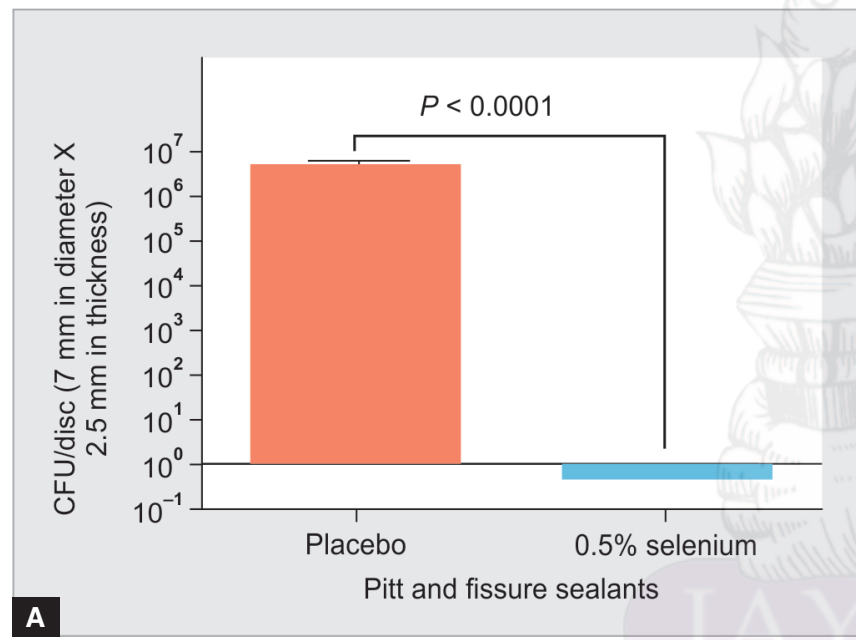

were partially lost (scores 2 and 3), while $1.0 \%$ were completely lost (score 4). With the UXT sealant, $5 \%$ were partially lost, while $14 \%$ were completely lost. DS and UXT did not significantly differ in clinical retention at 3,6 , or 9 month evaluations. There was no plaque growth around the DS-applied teeth, while in those same subjects $7 \%$ and $12 \%$ of the corresponding teeth with the UXT sealant had plaque growth around them after 9 and 12 months, respectively (Table 1). Those subjects with plaque growth around the UXT-sealed teeth, but having none around the DS treated teeth, were among those at high caries risk due to poor oral hygiene. The logistic regression test showed no association between any tested variable (gender, molar or premolar, upper or lower jaw, and left or right side) and sealant retention or plaque formation. There was neither adverse nor side effect reported throughout the study.

\section{Discussion}

The result of the in vitro phase of the present study (Graphs $1 \mathrm{~A}$ to $C$ ) was consistent with the report of a previous in vitro study that demonstrated the inhibition of biofilm formation on the surface and underneath organo-selenium sealants by two of the bacteria strains (S. mutans and S. salivarius) that were tested in the present
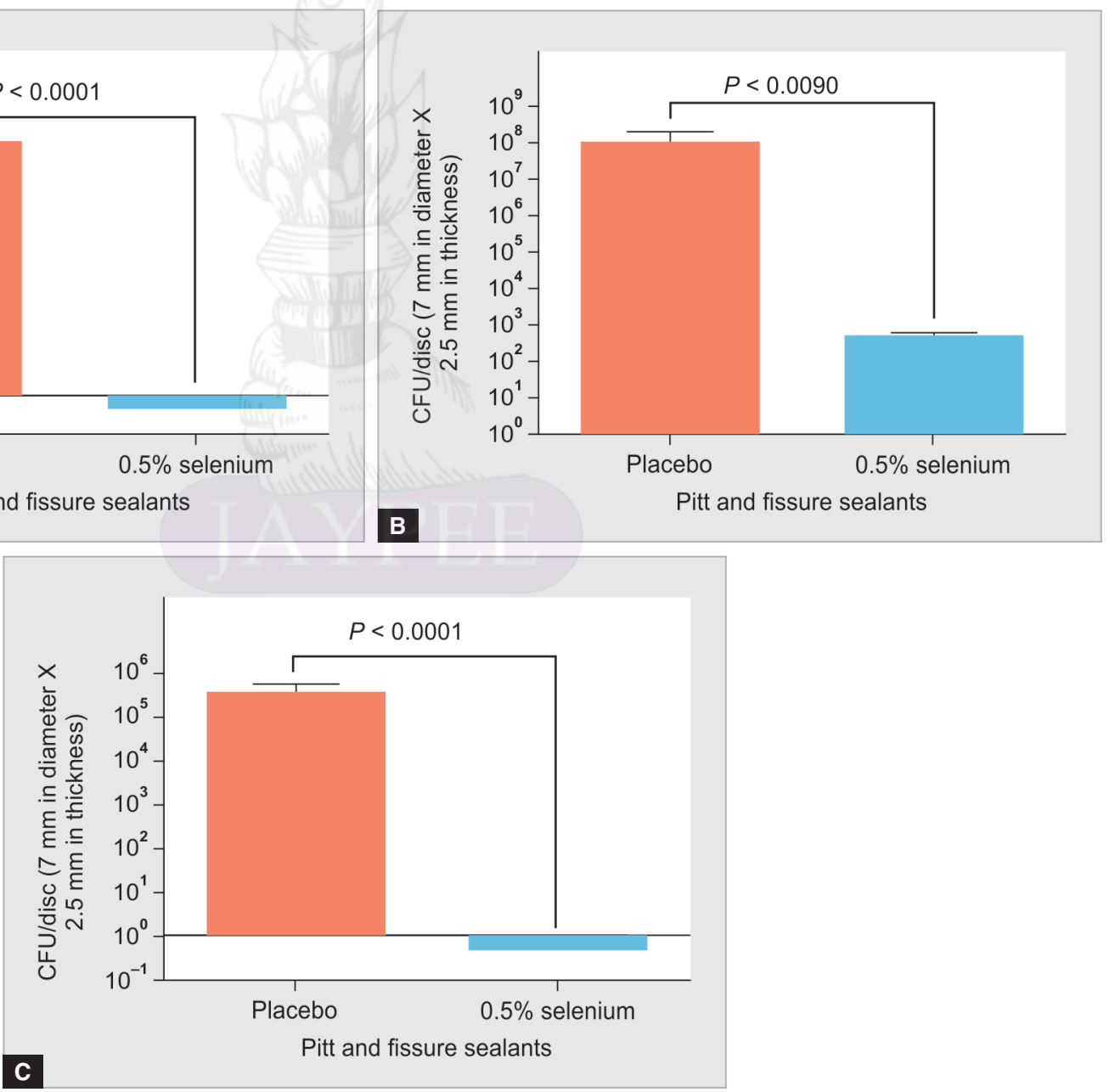

Graphs 1 A to C: (A) Graphic illustration of the inhibition of growth of S. salivarius bacteria by the DS pit and fissure sealant (which contains $0.5 \%$ selenium) and a placebo sealant. (B) Graphic illustration of the inhibition of growth of S. sanguis bacteria by the DS pit and fissure sealant (which contains $0.5 \%$ selenium) and a placebo sealant. (C) Graphic illustration of the inhibition of growth of S. mutans bacteria by the DS pit and fissure sealant (which contains $0.5 \%$ selenium) and a placebo sealant 
Table 1: Summary of the result of the clinical trial showing the clinical retention, mobility, caries formation, plaque growth, and adverse and side effects of the UXT and DS sealant

\begin{tabular}{|c|c|c|c|c|c|c|c|c|}
\hline \multirow[b]{2}{*}{ Condition assessed } & \multicolumn{2}{|l|}{3 months } & \multicolumn{2}{|l|}{6 months } & \multicolumn{2}{|l|}{9 months } & \multicolumn{2}{|l|}{12 months } \\
\hline & DS sealant & UXTPlus & DS sealant & UXTPlus & DS sealant & UXTPlus & DS sealant & UXTPlus \\
\hline Retention (\%) & 100 & 100 & 98.6 & 87.5 & 96.5 & 84.7 & $96.2^{*}$ & $80.9 *$ \\
\hline Mobility & 0 & 0 & 0 & 0 & 0 & 0 & 0 & 0 \\
\hline Caries formation & 0 & 0 & 0 & 0 & 0 & 0 & 0 & 0 \\
\hline Plaque growth & 0 & 0 & 0 & 0 & 0 & $7 \%$ & 0 & $12 \%$ \\
\hline Adverse effect & 0 & 0 & 0 & 0 & 0 & 0 & 0 & 0 \\
\hline Side effect & 0 & 0 & 0 & 0 & 0 & 0 & 0 & 0 \\
\hline
\end{tabular}

*Difference is statistically significant $(p=0.0004)$

study. ${ }^{16}$ What is clear from these findings is that bacteria that are known to participate in plaque formation on teeth are significantly inhibited in their ability to bind to the selenium-containing sealant, while these same bacteria show significant binding to the same sealant (placebo) without selenium.

The effectiveness of a resin-based pit and fissure sealant as a caries preventive device depends on the longevity of sealant coverage (i.e., clinical retention). However, caries can develop in the presence of a sealant due to plaque formation either around the edge of an improperly contoured sealant or underneath a shrunk sealant due to the ingress of saliva/bacteria under the sealant. With regard to this, the present study investigated and compared the ability of two resin-based pits and fissure sealants, selenium-containing (DS) and selenium-free (UltraSeal ${ }^{\mathrm{TM}}$ XT Plus), to exhibit an antimicrobial effect, preventing the growth of dental plaque and caries development around the sealant, while still maintaining an adequate clinical retention for a considerable period of time. The result of the study demonstrated that the DS sealant inhibited the growth of dental plaque in subjects where plaque growth was observed around the UXT sealant applied to the corresponding teeth on the opposite side of the dentition. This observation is attributed to the organo-selenium molecule component of the DS, which has been shown to serve as a catalytic generator of superoxide radicals $\left(\mathrm{O}_{2}{ }^{-}\right)$from the oxidation of thiols, ${ }^{23}$ an abundant element in human saliva. The superoxide radical is known to be toxic to different bacteria, such as Staphylococcus epidermidis, Staphylococcus aureus, Listeria monocytogenes, Salmonella typhimurium, and Escherichia coli in vitro. ${ }^{24-28}$ Thus, it is envisioned that, in the event of a sealant with microleakage with bacteria ingress underneath the sealant, the selenium-containing DS sealant would not allow bacteria to survive to initiate dental caries development and progression under the sealant.

It is pertinent to mention that effort was made to ensure that the subjects selected for the present study have the potential to develop dental plaque and caries lesions, by recruiting only children and adolescents at either moderate or high caries risk. The minimum qualification for participation was the presence of at least one factor that may increase caries risk. ${ }^{17}$ It was interesting to observe that the subjects that developed plaque around their UXT sealants were those at high caries risk due to poor oral hygiene, despite the fact that all subjects received instructions on good oral health behavior and were individually shown how to clean their teeth by trained oral health educators prior to the start of the treatment.

In this study, the presence of bacterial plaque was detected as red fluorescence along the edges of the sealant using QLF Pro ${ }^{\mathrm{TM}}$. HeinrichWeltzien et al. ${ }^{19}$ and Lennon et al. ${ }^{20}$ demonstrated that several strains of bacteria that are linked to caries and gingivitis produce metabolites that emit red fluorescence, especially in matured plaque ( $>1$ day), when illuminated with blue light emitted by QLF Pro ${ }^{\mathrm{TM}}$. This device uses this principle of green and red fluorescence imaging to detect and monitor the presence of bacterial plaque and, hence, bacterial activities on tooth surfaces as reported by de Josselin de Jong et al. ${ }^{21}$ and Van der Veen $\mathrm{MH}$ and de Josselin de Jong. ${ }^{22}$

In the present study, a significantly higher proportion of the DS sealant was retained than the UXT sealant after 12 months of application. The DS was retained in subjects where UXT failed and vice versa. Thus, retention or failure of either sealant did not follow any particular pattern, and logistic regression showed that it did not depend on the gender, type of tooth (molar or premolar), or position in dentition (upper or lower jaw and left or right side). Some people have the habit of using one side of the mouth more than the other side for food mastication, so to eliminate the possible influence of this factor on sealant retention, the positioning of the sealants was altered based on the computer output randomization number.

Adverse and side effects were monitored throughout the study, and none was reported nor clinically observed in the present study. We believed that this has to do with the fact that selenium does not leach out of the sealant material due to the covalent attachment of selenium to the polymer of the sealant. This property of non-leaching of selenium from the sealant enables the sealant to retain its antibacterial effect overtime, and this is an advantage of the organo-selenium dental sealants over fluoride-containing sealants, in which the gradual release of fluoride compromises the antibacterial effect of the fluoride-containing sealants over time. ${ }^{29-31}$ Fluoride-containing sealants would require the recharge of the sealant with more fluoride salt to enhance their antimicrobial activity.

It is important to mention that the present study has some limitations. The number of subjects (120 subjects) and the monitoring period ( 12 months) are limited for a clinical trial on pit and fissure sealants. The clinical trial part of the study is only one center study. A larger multicenter clinical trial with a large number of subjects with a minimum of 2 years follow-up is required for the results to be generalized.

\section{Conclusions}

The results of the clinical part of the present study demonstrated that the DS pit and fissure sealant completely prevented plaque growth around it with better clinical retention than the UXT sealant that offered only limited protection against plaque growth. This is consistent with the result of the in vitro phase of the study in which the DS sealant showed over 5 logs of inhibition of growth of three different strains of cariogenic bacteria. 


\section{ACKnowledgments}

This study was funded by SelenBio Inc., Austin, TX to Dr Bennett Amaechi through a contract with the University of Texas Health Science Center at San Antonio.

\section{References}

1. Brown LJ, Selwitz RH. The impact of recent changes in the epidemiology of dental caries on guidelines for the use of dental sealants. J Public Health Dent. 1995 Dec;55(5 Spec No):274-291.

2. Ahovuo-Saloranta A, Hiiri A, et al. Pit and fissure sealants for preventing dental decay in the permanent teeth of children and adolescents. Cochrane Database Syst Rev 2008 Oct;8(4):CD001830. DOI: 10.1002/14651858.CD001830.pub3.

3. Cueto El, Buonocore MG. Sealing of pits and fissures with an adhesive resin: its use in caries prevention. J Am Dent Assoc 1967 Jul;75(1):121-128.

4. Waggoner WF, Siegal M. Pit and fissure sealant application: updating the technique. J Am Dent Assoc 1996 Mar;127(3):351-361, quiz 391-392.

5. Nicholson JW. Polyacid-modified composite resins ("compomers") and their use in clinical dentistry. Dent Mater 2007 May;23(5):615-622. DOI: 10.1016/j.dental.2006.05.002.

6. Ruse ND. What is a "compomer"? J Can Dent Assoc 1999 Oct;65(9): 500-504.

7. Llodra JC, Bravo M, et al. Factors influencing the effectiveness of sealants - a meta-analysis. Community Dent Oral Epidemiol 1993 Oct;21(5):261-268.

8. Mejare I, Lingstrom P, et al. Caries-preventive effect of fissure sealants: a systematic review. Acta Odontol Scand 2003 Dec;61(6):321-330.

9. Ripa LW. Sealants revisted: an update of the effectiveness of pit-andfissure sealants. Caries Res 1993 Feb;27 Suppl 1:77-82.

10. Carlsson A, Petersson M, et al. 2-year clinical performance of a fluoride-containing fissure sealant in young schoolchildren at caries risk. Am J Dent 1997 Jun;10(3):115-119.

11. Handelman SL. Effect of sealant placement on occlusal caries progression. Clin Prev Dent 1982 Sep-Oct;4(5):11-16.

12. Mertz-Fairhurst EJ, Schuster GS, et al. Arresting caries by sealants: results of a clinical study. J Am Dent Assoc 1986 Feb;112(2):194-197.

13. Griffin SO, Oong E, et al. The effectiveness of sealants in managing caries lesions. J Dent Res 2008 Feb;87(2):169-174. DOI: 10.1177/154405910808700211.

14. Mickenautsch S, Yengopal V. Validity of sealant retention as surrogate for caries prevention - a systematic review. PLoS One 2013 Oct 23;8(10):e77103. DOI: 10.1371/journal.pone.0077103.

15. Hammond A, Dertien J, et al. Serum inhibits P. aeruginosa biofilm formation on plastic surfaces and intravenous catheters. J Surg Res 2010 Apr;159(2):735-746. DOI: 10.1016/j.jss.2008.09.003.
16. Tran $P$, Hamood A, et al. Organo-selenium-containing dental sealant inhibits bacterial biofilm. J Dent Res 2013 May;92(5):461-466. DOI: 10.1177/0022034513482141.

17. American Dental Association Council on Scientific Affairs. Professionally applied topical fluoride: evidence-based clinical recommendations. J Am Dent Assoc 2006 Aug;137(8):1151-1159.

18. Govoni M. Success with pit and fissure sealants. Dent Assist 2002 May-Jun;71(3):8-9.

19. Heinrich-Weltzien R, Kuhnisch J, et al. Quantitative light-induced fluorescence (QLF) - a potential method for the dental practitioner. Quintessence Int 2003 Mar;34(3):181-188.

20. Lennon AM, Buchalla W, et al. The ability of selected oral microorganisms to emit red fluorescence. Caries Res 2006;40(1):2-5.

21. de Josselin de Jong $E$, Hall AF, et al. Quantitative light-induced fluorescence detection method. A Monte Carlo simulation model. In: Stookey GK, ed. Early Detection of Dental Caries: Proceedings of the 1st Annual Indiana Conference. Indianapolis, IN: Indiana University Press, 1996. pp. 91-104.

22. Van der Veen $\mathbf{M H}$, de Josselin de Jong E. Application of quantitative light-induced fluorescence for assessing early caries lesions. In: Faller RV, ed. Assessment of Oral Health: Diagnostic Techniques and Validation Criteria. Monogr Oral Sci. Basel:Karger; 2000. 17: pp. 144-162.

23. Seko $Y$, Imura N. Active oxygen generation as a possible mechanism of selenium toxicity. Biomed Environ Sci 1997 Sep;10(2-3):333-339.

24. Babior BM, Curnutte JT, et al. Biological defense mechanisms. Evidence for the participation of superoxide in bacterial killing by xanthine oxidase. J Lab Clin Med 1975 Feb;85(2):235-244.

25. Bortolussi R, Vandenbroucke-Grauls CM, et al. Relationship of bacterial growth phase to killing of Listeria monocytogenes by oxidative agents generated by neutrophils and enzyme systems. Infect Immun 1987 Dec;55(12):3197-3203.

26. Hoepelman IM, Bezemer WA, et al. Bacterial iron enhances oxygen radical-mediated killing of Staphylococcus aureus by phagocytes. Infect Immun 1990 Jan;58(1):26-31.

27. Kramer GF, Ames BN. Mechanisms of mutagenicity and toxicity of sodium selenite $\left(\mathrm{Na}_{2} \mathrm{SeO}_{3}\right)$ in Salmonella typhimurium. Mutat Res 1988 Sep;201(1):169-180.

28. Rosen H, Klebanoff SJ. Role of iron and ethylenediaminetetraacetic acid in the bactericidal activity of a superoxide anion-generating system. Arch Biochem Biophys 1981 May;208(2):512-519.

29. Loyola-Rodriguez JP, Garcia-Godoy F. Antibacterial activity of fluoride release sealants on mutans streptococci. J Clin Pediatr Dent 1996 Winter;20(2):109-111.

30. Matalon S, Slutzky H, et al. Surface antibacterial properties of fissure sealants. Pediatr Dent 2003 Jan-Feb;25(1):43-48.

31. Naorungroj $\mathrm{S}$, Wei $\mathrm{HH}$, et al. Antibacterial surface properties of fluoride-containing resin-based sealants. J Dent 2010 May;38(5): 387-391. DOI: 10.1016/j.jdent.2010.01.005. 